\title{
Blockchain technology and its applications to combat COVID-19 pandemic
}

\author{
Abhishek Sharma ${ }^{1} \cdot$ Shashi Bahl ${ }^{2}$ (D) $\cdot$ Ashok Kumar Bagha $^{1} \cdot$ Mohd Javaid $^{3} \cdot$ Dinesh Kumar Shukla $^{1} \cdot$ Abid Haleem $^{3}$
}

Received: 27 May 2020 / Accepted: 15 October 2020 / Published online: 22 October 2020

(C) Sociedade Brasileira de Engenharia Biomedica 2020

\begin{abstract}
Background and aims The world is currently facing multifaceted problems due to the emergence of the COVID-19 (coronavirus) pandemic. Blockchain technology (BT) plays a vital role in creating a platform for adequately managing the COVID-19 pandemic. Methods The latest information on the blockchain and its application in solving challenging problems due to the COVID-19 pandemic are collected and discussed from the available literature searched through PubMed, Scopus, and Google scholar. Results In this paper, we propose a blockchain-based platform to combat this pandemic. Furthermore, we have identified and discussed nine significant applications of blockchain in solving the problem arising from the COVID-19 pandemic.

Conclusions The severity of COVID-19 was so tremendous that the World Health Organization (WHO) had to declare it as a pandemic within a month of its full-scale expansion. The greatest challenge most governments are suffering from is the lack of a precise mechanism to detect the newly infected cases and predict coronavirus infection risk. So, we need a technologyempowered solution to fight during this COVID-19 crisis. The various features of blockchain technology, such as decentralization, transparency, and immutability, can help control this pandemic by early detection of outbreaks, fast-tracking drug delivery, and protecting user privacy during treatment.
\end{abstract}

Keywords Blockchain technology (BT) · COVID-19 · Coronavirus · Healthcare

\section{Introduction}

In this worldwide health crisis, the medical industry is looking for newer technologies to monitor and control the COVID-19

\section{Highlights}

- Blockchain technology (BT) provides a better platform for effective management of the COVID-19 pandemic.

- Blockchain technology helps create decentralization, transparency, immutability, and early detection of infected cases.

- Blockchain technology is useful for disease control, patient traceability, and improved management of the healthcare system.

Shashi Bahl

shashi.bahl@ptu.ac.in

Abhishek Sharma

158abhisharma@gmail.com

Ashok Kumar Bagha

baghaak@nitj.ac.in

Mohd Javaid

mohdjavaid0786@gmail.com

Dinesh Kumar Shukla

shukladk@nitj.ac.in (coronavirus) pandemic. Thus, to monitor and control the spread of any virus, accurate and trustable data are required or essential. However, in the present scenario, the existing technology lacks the trustable data that may provide the

Abid Haleem

haleem.abid@gmail.com

1 Department of Mechanical Engineering, Dr B.R. Ambedkar National Institute of Technology, Jalandhar 144011, India

2 Department of Mechanical Engineering, I.K. Gujral Punjab Technical University Hoshiarpur Campus, Hoshiarpur 146001, India

Department of Mechanical Engineering, Jamia Millia Islamia, New Delhi 110025, India 
correct information about the novel coronavirus widespread or outbreak. The sources such as public hospitals and clinical laboratories can provide information about the COVID-19 pandemic patients, but the data may not be faithful because it is not monitored and appropriately stored and possibly not collected according to the set guidelines (Bansal et al. 2020; Haleem et al. 2020; Ting et al. 2020).

To solve these kinds of issues, blockchain technology (BT) can play a vital role in tracking the spread of the coronavirus easily, identify high-risk patients, and highly competent to control the infection in real-time defined as a digital database that contains information that can be simultaneously used and shared within an extensive decentralized and publicly accessible network. The use of telemedicine for diabetic patients in fighting the COVID-19 pandemic has already been demonstrated (Bahl et al. 2020; Ghosh et al. 2020; Vaishya et al. 2020). Various technologies such as industry 4.0, biosensors, 3D scanning, and multi-agent system (Bahl et al. 2020; Haleem et al. 2020; Javaid et al. 2020; Sharma et al. 2020) can be gainfully employed to identify the infected cases .

Blockchain technology is a digital ledger that consists of distributed, decentralized, and often-times public data. The blockchain technology has mainly three parts such as blocks, miners, and nodes. The chains consist of multiple blocks, and each block takes the information about the data, nonce, and the hash. The miners can create a new block of chains by using a process called mining. Nodes are the electronic devices that maintain a copy of the blockchain and keep its network functioning (Chang and Park 2020; Imtyaz et al. 2020; Khan et al. 2020). Figure 1 represents the role of blockchain technology in the treatment or collection of data about active patients during the COVID-19 pandemic. Here, the blockchain participating nodes are used for patients, testing and clinical laboratories, hospitals, and government sites. Besides, the documents on the digital ledger are patient records, sample test results, treatment status, and discharge summary. Furthermore, it is clear that the blockchain technology processes involve the following steps:

(a) Collecting requisite data from the blockchain participating nodes, and

(b) Creating raw data and subsequently developing it into a big scale data.

These steps establish the online digital document ledger. Blockchain technology assures the security of the collected data and helps to maintain its privacy. The blockchain secured data are analyzed by using various artificial intelligence-based solutions. BT provides various feasible solutions for the COVID-19 pandemic, such as outbreak tracking, donation tracking, and medical supply chain management. It is also used to establish fast, preserving, and reliable data exchange with the stakeholders. Worldwide, hospitals and masses face the shortage of medical equipment to fight this pandemic (Iyengar et al. 2020, 2020; Suman et al. 2020).
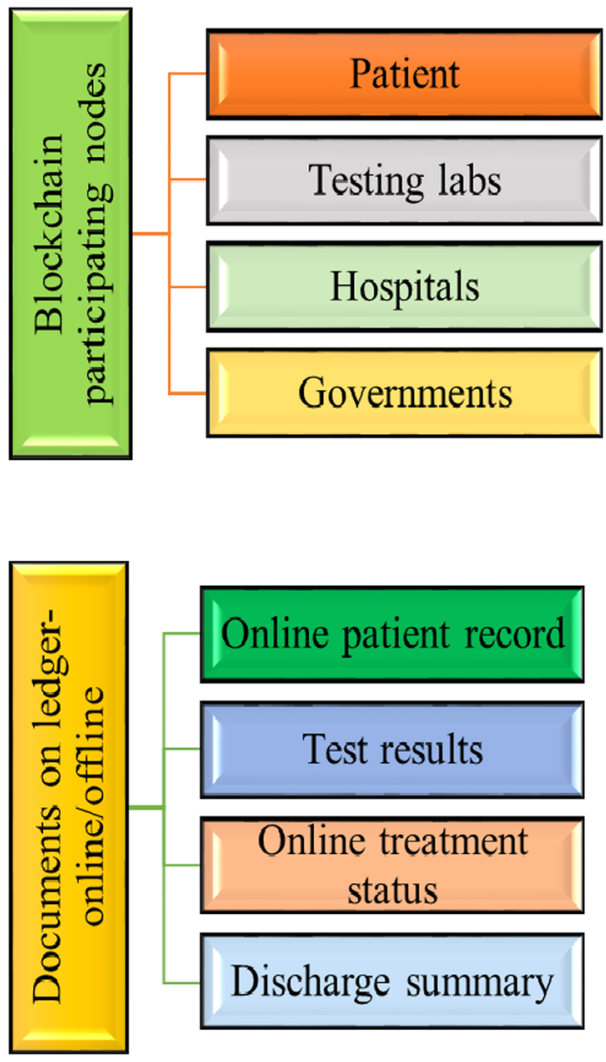

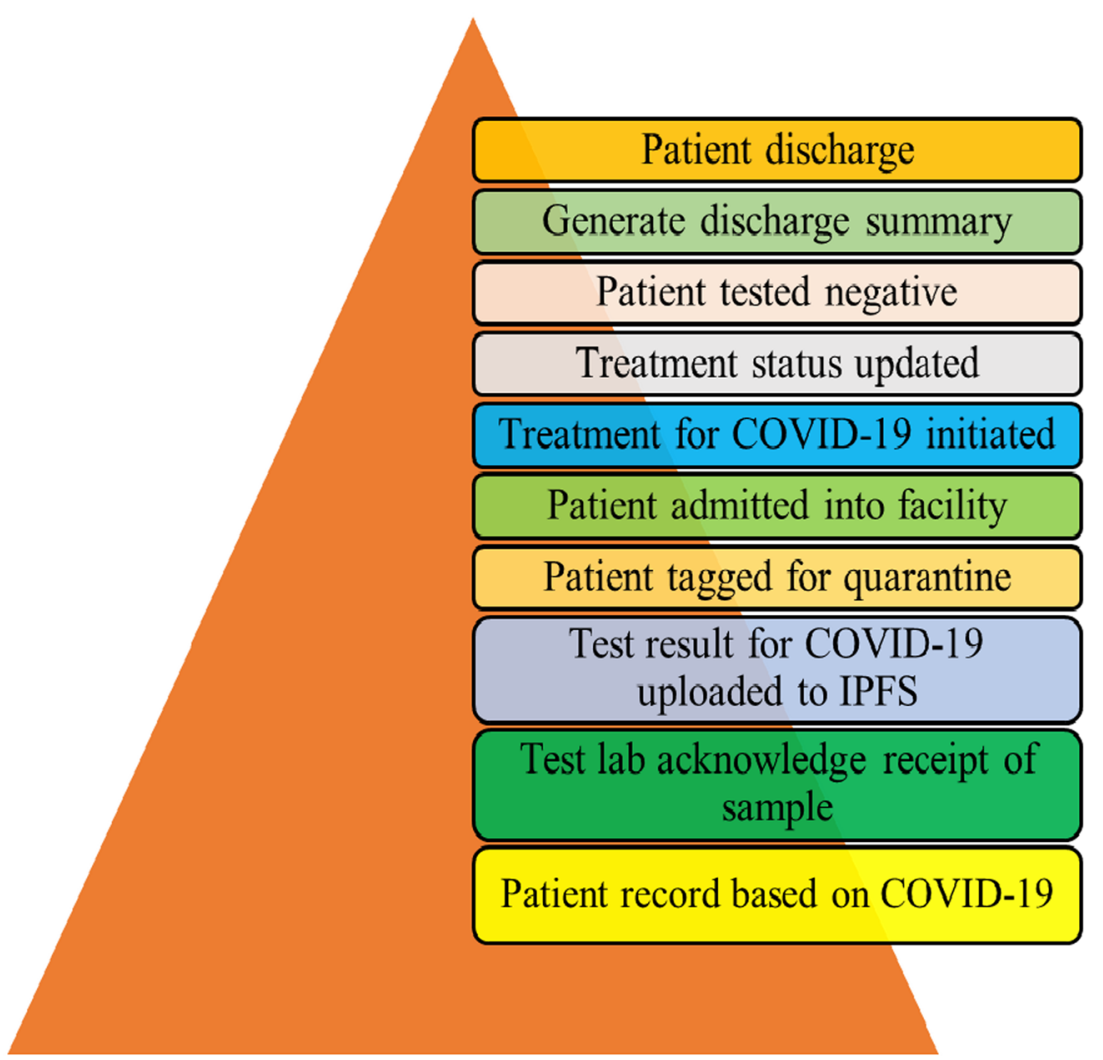

Fig. 1 Role of blockchain technology for COVID-19 pandemic 


\section{Significant benefits of blockchain technology for COVID-19 pandemic}

Blockchain technology enables distributed, encrypted, and secure logging of digital transactions. It is expected to revolutionize computing in several areas, mainly where centralization is unnatural and privacy is essential. It can be leveraged globally to track the spread of the coronavirus infection by deploying a blockchain network on citizens' mobile devices. One of the major potentials of blockchain technology is to preserve the patient information. It can simplify the fasttracking of drug trials and record and track all fund raising activities and donations transparently (Khurshid 2020; Mishra et al. 2020; Resiere et al. 2020). So, outbreak tracking, user privacy protection, medical supply chain management, and donation tracking are the various areas in which blockchain technology can play a vital role in fighting against the COVID-19 crisis (De' et al. 2020; Jaly et al. 2020; Singh et al. 2020; Vaishya et al. 2020).

\section{Digitally data storage of COVID-19 patients using blockchain technology}

One of the main issues during this pandemic time is the need for reliable and up-to-date data concerning the outbreak and spread of novel coronavirus. Blockchain technology can help to resolve this problem very effectively. One of the essential advantages of this technology is providing verifiable and secure data by using its distributed ledger technology and peer-to-peer networking features (Garg et al. 2020; Rab et al. 2020; Shams et al. 2020). During this pandemic, this technology is instrumental in recording patient information with COVID-19 symptoms, locations, and history of health conditions with high privacy. Many platforms have recently been launched which use this technology to facilitate sharing the information and valuable data related to COVID-19. The World Health Organization (WHO) has launched MiPasa in March 2020. It is a blockchain technologybased platform which facilitates the fully private information sharing between individuals, state authorities, and health institutions. This platform also allows privacy-enabled self-reporting by allowing public health officials and individuals to upload data about different infection times and exact location (Govindan et al. 2020; Singh et al. 2020).

\section{Blockchain technology for public surveillance system during COVID-19 pandemic}

Blockchain technology can successfully track the patient's moment and provide real-time data about the affected areas and direct fighting efforts. This technology is also useful for tracking of person movement in a virus-free zone. The government and health organizations can monitor potential patients at every stage by using blockchain technology with reliability and accuracy (Papadopoulos et al. 2020; Pratap Singh et al. 2020). Information on safe zones such as population, location, and current coronavirus outbreak status is recorded using a chain of blocks in which each block can store update of the outbreak at a particular time (Christodoulou et al. 2020; Vaishya et al. 2020). Combining blockchain technology with artificial intelligence (AI) and geographical information system (GIS) can make the public surveillance system more effective and robust.

\section{Process of blockchain for COVID-19 pandemic}

The blockchain technology participating nodes are used for patients, testing and clinical laboratories, hospitals, and government sites. Besides, the documents on the digital ledger are patient records, sample test results, treatment status, and discharge summary.

Figure 1 shows the essential steps used in blockchain technology to track and record the data's COVID-19 active patients. Initially, the patient is examined and diagnosed according to the pre-symptoms of COVID-19 disease. The patient sample is taken, and if it is found positive, then the patient is quarantined for at least 14 days. During this period, the blockchain technology application is used to do the treatment and the monitoring of the patient. Then, the recovery phase starts, and the patient is re-examined for COVID-19. However, if the sample result is negative, the patient is discharged, and a discharge summary is generated. The patient data are recorded for future reference; its privacy is maintained and produced when needed to show on a large scale. The blockchain technology gives assurance about the accuracy of the patient's recorded data.

According to the Organization of Economic Cooperation and Development (OECD) (OECD Economic Outlook, Interim Report March 2020: Coronavirus: the world economy at risk, 2020), the global economy has been slowed down and has grown at its slowest rate since 2009 due to the complete shutdown of various sectors such as supply chain, insurance, tourism, agriculture, construction, and automobiles.

\section{Some cases of blockchain for COVID-19 pandemic}

Some cases in which blockchain technology has been used to combat the COVID-19 pandemic are discussed subsequently:

Hashlog This was the first project created by the Georgiabased health technology startup Acoer (Hashgraph 2020). Hashlog blockchain solution can be enabled by distributed 
blockchain ledger technology, which ensures logging and data visualization of coronavirus outbreak from US centers' public data for disease control (CDC) and WHO.

VeChain It is a blockchain-based platform built to monitor vaccine production in China (Nasdaq 2018). All activities related to vaccine manufacturing, from materials, codes to package, are recorded and stored on distributed ledgers. It also provides an effective method to reduce the risk of potential modifications to vaccine information.

PHBC This blockchain-based platform is used for continual and anonymous verification of communities and workplaces that are free from coronavirus COVID-19 and other high-risk viruses to aid them in staying free of deadly diseases. An essential feature of this blockchain technology-based platform is its ability to trace uninfected persons' movement and restrict their return if they have gone to the infected areas.

Hyperchain It is a donation-based platform developed for supporting governments and health organizations in the donation process to infected patients in China. Hyperchain can connect up to millions of nodes so that many users can reach to donated goods and necessary medical equipment from the factories to solve the facilities shortage issues during this pandemic period.

\section{Main applications of blockchain technology in the COVID-19 pandemic}

\section{Disease control}

For infectious disease control and preventing the spread of the pandemic, effective and accurate disease surveillance is necessary (ZIGURAT: Innovation and Technology Business School 2020). Ebola virus, yellow fever, cholera in Africa, Nipah in Asia (Sharma 2020), Middle East respiratory syndrome, coronavirus, etc., are the diseases that can be monitored and controlled using blockchain technology. It can be used globally to track the spread of COVID-19 infections among humans and is carried out by deploying the blockchain network in-country citizens' electronic devices. In the COVID-19 pandemic, blockchain technology is essential to support the virus victims by recording immutably patient's symptoms of infection.

\section{Traceability}

Traceability indicates the tracking of infected patients. It is imperative to control the spread of coronavirus. With blockchain technology, one can track the infected patient's movements, provide real-time data about affected areas, and report direct fighting efforts. Blockchain technology is also useful to implement tracking of person movements in virusfree zones (Juma et al. 2019). Information on safe zones such as population, location, and current coronavirus outbreak status is recorded using the chain blocks. Goods and medical supplies need to be continuously tracked to ensure transparency in the medical supply chain. This tracing can be done by transaction recording and monitoring capabilities of the blockchain network.

\section{Bolstering supply chain of medical parts during this crisis}

In this pandemic crisis, maintaining a continuous supply of medicines and foods has become a considerable challenge for the healthcare sector. Blockchain technology has proved very useful in the goods supply chain and trading supply chain (Gonczol et al. 2020). It can ensure reliability for the medical chain by secure linking between blocks and transactions. Thus, to maintain privacy in data of supply chain, blockchain encryption is used. Recently, IBM launched a blockchain network to bolster the medical supply chain during COVID-19, under the project name "Rapid Supplier Connect."

\section{Improving transparency during the treatment of infected patients}

Transparency is one of the most important features of blockchain technology. It is indispensable to secure the personal data and information related to the patients under treatments. The outbreak of fake news on social platforms generates fear and panic due to unverified data. Blockchain can provide a potential way to ensure the scrutiny of data accuracy by its ability to validate the information and real-time data updates. It can facilitate the transition from institution driven interoperability to patient-centered interoperability (Dimitrov 2019).

\section{Tracking of healthcare instruments}

Blockchain technology can play a vital role in tracking healthcare instruments during the COVID-19 pandemic. It ensures the safe and secure movement of healthcare instruments from one place to another. Recently, Alipay, along with the Zhejiang Provincial Health commission and the Economy and Information Technology department China, has launched a blockchain technology-based platform (Powers 2020). It consists of recording and tracking the "coronavirus pandemic fighting materials" such as masks, gloves, and protection gears. 


\section{Improving the recovery of infected patients}

Infected patient's recovery rate can be improved if they get the treatment at the right time. Blockchain technology helps to monitor the quarantine cases effectively at home and the hospitals. It enables the drug supply faster, which will play an important role in the recovery of infected patients. In the COVID-19 pandemic, blockchain can record patient symptoms, location, and historical health conditions with high privacy. The data block is decentralized over the distributed networks of governments, healthcare professionals, and endusers (Duggal 2020).

\section{Storage and transfer of treatment-related information}

Storage of collected data and transferring the treatment-related information are one of the most critical and challenging tasks during this COVID-19 pandemic. Between the years 2009 to 2017, more than 176 million patients' records were exposed to data breaches. Blockchain technology can keep an incorruptible, decentralized, and transparent log of patient data. Blockchain technology allows patients, doctors, and healthcare providers to share the same information quickly and safely (Kuo 2020).

\section{Better healthcare protection}

Blockchain technology powered the platforms that tackle the affordable and quality healthcare protection (Helms 2020). It can provide a feasible solution for tracking the coronavirus outbreak to protect more patients from this pandemic. It enables location tracking of infected patients through real-time routine checking to get the proper treatment at the right time. If medical professionals and hospitals had a secure and reliable database of the health record, it would reduce the risk of misdiagnosis.

\section{Effective healthcare management during the crisis}

A well-designed healthcare management system is necessary to deal with such a big crisis like COVID-19 in the present and for the future. According to the WHO, every month, frontline health responders need more than 89 million masks, 39 million gowns, 76 million gloves, and 2.9 million liters of hand sanitizer to protect themselves and others from COVID-19. Blockchain technology helps to manage the supply of all the above-mentioned items (Lacina 2020). It is further expected to improve the medical record management, insurance claim process, clinical and biomedical research, and advance biomedical and healthcare ledger during this COVID-19 crisis (Kuo et al. 2017).

\section{Significant contributions of blockchain for COVID-19 pandemic}

Figure 2 explains contributions of blockchain technology that are useful during this COVID-19 pandemic. This pandemic has affected sectors like healthcare, finance, politics, economics, and education. Blockchain technology can play a vital role in the effective management of the post COVID-19 world. Blockchain technology's key features can support the proper implementation of many use cases such as contact tracing, disaster relief, patient information sharing, e-government, supply chain management, immigration management, manufacturing management, automated surveillance, contactless delivery, and online education.

When a person is diagnosed with COVID-19, it is necessary to identify people who happen to have close interactions or contacts with the patient during the infectious incubation period. It can be made possible through contact tracing, which aims to discover the social interactions that the patient had during this period. Various applications have been developed for this purpose using bluetooth low energy (BLE) technology that records close encounters between mobile phones, wearables, and the internet of things (IoT). Data security and privacy for users are the significant challenges in contact tracing because they are stored in a centralized cloud and may lead to loss of user's ownership of their data. Thus, blockchain technology can play a vital role in providing security and privacy to users' data as it offers a decentralized solution wherein users can have full control over their data. Hence, the patient's privacy can be taken care of by enabling pseudo-anonymity.
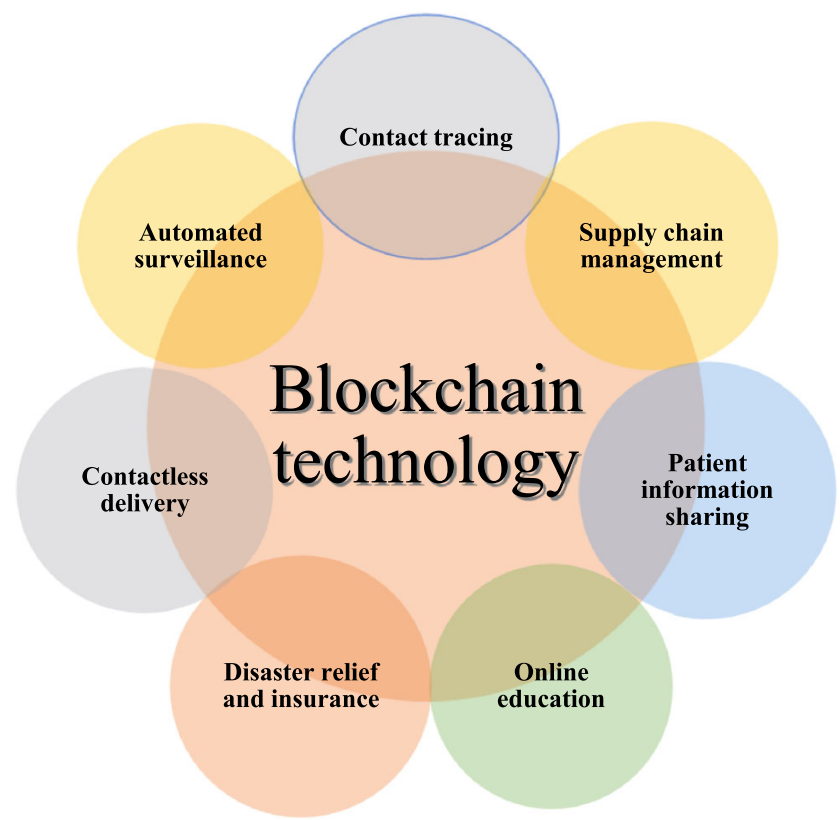

Fig. 2 Contributions of blockchain technology during COVID-19 pandemic 
The COVID-19 pandemic has affected the global supply chain badly. It is becoming challenging to tackle the losses suffered during the lockdown and the difficulties that the factories are facing in creating a new kind of working environment that includes social distancing and working with a minimum number of people to avoid physical contact. It has led to a crisis in supply and demand. Medical equipment and pharmaceutical supply chains face difficulty maintaining an unbroken chain because of the rise in demand. Also, panic buying is one of the primary reasons for the rise in demand for certain products such as groceries. Blockchain technology can help in linking stakeholders and developing chains that have provenance and transparency. This can also help make smart contracts that can provide a high level of access restrictions and automation.

On one hand, it is important to share relevant data with the healthcare collaborators nationally and internationally to avail strong datasets for COVID-19 research. On the other hand, it is vital to ensure that patient's privacy is taken care of and sharing mechanism does not violate national and international data-sharing regulations. Detailed information related to blood oxygen level, medication, etc., can be collected from medical IoT devices. Blockchain can help in real-time data sharing and make data sharing between hospitals and medical professionals easier. Adopting blockchain can help in resolving problems related to data forging and mutation. Blockchain involves decentralized storage. Therefore, security and privacy of health-related data can be improved, thus maintaining trust between stakeholders.

During this pandemic, all colleges and universities have been closed for an unpredictable duration, which badly affected the education system. Online education is the only solution to combat these situations.

However, there are many challenges in front of colleges and schools to provide effective online education. Secure tracking of the learning process, difficulty in verifying the student's credentials, like degree certificate, real evaluation of student's performance, and threats related to data security are significant challenges in front of everyone. Blockchainbased platforms enable secure cross-platform sharing of online content and provide a secure environment for both students and teachers' data. The world economy and various businesses have been affected badly because of lockdowns and social distancing rules throughout the world. Governments and financial organizations' responsibility is to provide the necessary aid to businesses by providing loans. Using old paper-based techniques of providing loans and other financial services will consume much time. So, blockchain with smart contracts can provide the solution for this problem. It will simplify the application and approval process for the loan and insurance-related services.

Thus, to fight with novel coronavirus (COVID-19) disease, it is essential to take some necessary steps such as social distancing, use face masks, use protection shield, and continuously assess symptoms like fever and cough. However, these steps are not habitual for the human being. So, a certain level of continuous and automated surveillance is required for alerts to save humans' lives. Furthermore, there are many areas where the transmission rate of COVID-19 is very high, so contactless delivery is required to ensure foods and medicine demands. Blockchain technology-based UAVs and robots can be used for automated surveillance and contactless delivery. Blockchain-powered UAVs and robots can effectively work without any human intervention in high-rate transmission zones.

\section{Limitation of blockchain technology}

It is observed that BT has the following limitations, such as:

(i) It involves enormous energy consumption because it requires powerful hardware resources for each transaction.

(ii) Scalability is the major limitation of this technology. It is because validation requires some time due to the authorization of transactions by the majority of nodes.

(iii) The complexity of blockchain and the requirement of an extensive network of users are another disadvantage of this technology.

(iv) Privacy preservation is another big challenge in front of this technology.

\section{Future development of blockchain technology}

Blockchain technology should be improved in such a way so that network latency is reduced, and a more secure environment is created for storing and transmission of critical information. Lightweight blockchain design in healthcare is necessary to optimize data verification and transaction communication. Building customized ledgers that can be placed on local servers in the outbreak area enhances blockchain performance. In the future, blockchain technology with other emerging technologies, such as artificial intelligence (AI), big data, and Cloud computing, can provide a very effective way to handle deadly pandemic like coronavirus. Recently, Taiwan leveraged big data combined with $\mathrm{AI}$ to control the virus spread (Waltz 2020). China has recently exploited the mobility of drones (Chi-Nguyen et al. 2018) to improve the provisions of medical supplies. Alibaba has also integrated AI with Cloud computing for supporting coronavirus data analytics (Yu 2020). 


\section{Conclusion}

In this study, we have presented the utilization of blockchain technology to combat the COVID-19 pandemic. The critical role of blockchain technology is to help control the spread of this pandemic. This technology can help during this pandemic crisis by providing improved solutions, outbreak tracking, user privacy protection, the performance of the medical supply chain, donation tracking, and safe day-to-day operations. Blockchain technology should be applied in such a way that network latency can be reduced with a secure environment for storing and transmission of critical information. The ultimate combination of blockchain technology with other emerging technologies, such as artificial intelligence, big data, and Cloud computing, can effectively handle deadly pandemic like coronavirus.

\section{Compliance with ethical standards}

Conflict of interest The authors declare that they do not have any conflict of interest regarding this article.

\section{References}

Bahl S, Javaid M, Bagha AK, Singh RP, Haleem A, Vaishya R, et al. Biosensors applications in fighting COVID-19 pandemic. Apollo Med. 2020;17:221-3. https://doi.org/10.4103/am.am_56_20.

Bahl S, Singh RP, Javaid M, Khan IH, Vaishya R, Suman R. Telemedicine technologies for confronting COVID-19 pandemic: a review. Journal of Industrial Integration and Management: Innovation and Entrepreneurship. 2020. https://doi.org/10.1142/ S2424862220300057.

Bansal A, Garg C, Padappayil RP. Optimizing the implementation of COVID-19 "immunity certificates" using blockchain. J Med Syst. 2020;44:140. https://doi.org/10.1007/s10916-020-01616-4.

Chang MC, Park D. How can blockchain help people in the event of pandemics such as the COVID-19? J Med Syst. 2020;44:102. https://doi.org/10.1007/s10916-020-01577-8.

Chi-Nguyen D, Pathirana PN, Ding M, Seneviratne A. Secrecy performance of the UAV enabled cognitive relay network. 2018 IEEE 3rd International Conference on Communication and Information Systems (ICCIS), 2018, p. 117-21. doi: https://doi.org/10.1109/ ICOMIS.2018.8644982.

Christodoulou K, Christodoulou P, Zinonos Z, Carayannis EG, Chatzichristofis SA. Health information exchange with blockchain amid COVID-19-like pandemics. 2020 16th International Conference on Distributed Computing in Sensor Systems (DCOSS), 2020, p. 412-7. doi: https://doi.org/10.1109/ DCOSS49796.2020.00071.

De’ R, Pandey N, Pal A. Impact of digital surge during Covid-19 pandemic: a viewpoint on research and practice. Int J Inf Manag. 2020;55:102171. https://doi.org/10.1016/j.ijinfomgt.2020.102171.

Dimitrov DV. Blockchain applications for healthcare data management. Healthc Inform Res. 2019;25:51-6. https://doi.org/10.4258/hir. 2019.25.1.51.

Duggal M. Blockchain and AI amidst the coronavirus crisis: 'a call to arms.' CryptoNewZ. 2020. [cited 2020 October 1]. Available from: https://www.cryptonewsz.com/blockchain-and-ai-amidst-thecoronavirus-crisis-a-call-to-arms/.
Garg C, Bansal A, Padappayil RP. COVID-19: prolonged social distancing implementation strategy using blockchain-based movement passes. J Med Syst. 2020;44:165. https://doi.org/10.1007/s10916020-01628-0.

Ghosh A, Gupta R, Misra A. Telemedicine for diabetes care in India during COVID19 pandemic and national lockdown period: guidelines for physicians. Diabetes Metab Syndr Clin Res Rev. 2020;14: 273-6. https://doi.org/10.1016/j.dsx.2020.04.001.

Gonczol P, Katsikouli P, Herskind L, Dragoni N. Blockchain implementations and use cases for supply chains - a survey. IEEE Access. 2020;8:11856-71. https://doi.org/10.1109/ACCESS.2020. 2964880.

Govindan K, Mina H, Alavi B. A decision support system for demand management in healthcare supply chains considering the epidemic outbreaks: a case study of coronavirus disease 2019 (COVID-19). Transport Res E-Log. 2020;138:101967. https://doi.org/10.1016/j. tre.2020.101967.

Haleem A, Gupta P, Bahl S, Javaid M, Kumar L. 3D scanning of a carburetor body using COMET 3D scanner supported by COLIN 3D software: issues and solutions. Mat Today Proc. 2020. https:// doi.org/10.1016/j.matpr.2020.07.427.

Haleem A, Javaid M, Vaishya R, Deshmukh SG. Areas of academic research with the impact of COVID-19. Am J Emerg Med. 2020;38:1524-6. https://doi.org/10.1016/j.ajem.2020.04.022.

Hashgraph $\mathrm{H}$. Acoer coronavirus tracker, powered by Hedera Hashgraph, now freely available to general public with added clinical trial data. 2020. Available from: https://www.hedera.com/blog/acoercoronavirus-tracker-powered-by-hedera-hashgraph-now-freelyavailable-to-general-public-with-added-clinical-trial-data.

Helms K. Coronavirus relief: cryptocurrency aid programs launched to combat Covid-19 outbreak. BitcoinCom. 2020. [cited 2020 March 27]. Available from: https://news.bitcoin.com/coronavirusrelief-aid-cryptocurrency/.

Imtyaz A, Haleem A, Javaid M. Analysing governmental response to the COVID-19 pandemic. J Oral Biol Craniof Res. 2020;10:504-13. https://doi.org/10.1016/j.jobcr.2020.08.005.

Iyengar K, Bahl S, Vaishya R, Vaish A. Challenges and solutions in meeting up the urgent requirement of ventilators for COVID-19 patients. Diabetes Metab Syndr Clin Res Rev. 2020;14:499-501. https://doi.org/10.1016/j.dsx.2020.04.048.

Iyengar KP, Vaishya R, Bahl S, Vaish A. Impact of the coronavirus pandemic on the supply chain in healthcare. Br J Healthc Manag. 2020;26:1-4. https://doi.org/10.12968/bjhc.2020.0047.

Jaly I, Iyengar K, Bahl S, Hughes T, Vaishya R. Redefining diabetic foot disease management service during COVID-19 pandemic. Diabetes Metab Syndr Clin Res Rev. 2020;14:833-8. https://doi.org/10.1016/ j.dsx.2020.06.023.

Javaid M, Haleem A, Vaishya R, Bahl S, Suman R, Vaish A. Industry 4.0 technologies and their applications in fighting COVID-19 pandemic. Diabetes Metab Syndr Clin Res Rev. 2020;14:419-22. https:// doi.org/10.1016/j.dsx.2020.04.032.

Juma H, Shaalan K, Kamel I. A survey on using blockchain in trade supply chain solutions. IEEE Access. 2019;7:184115-32. https:// doi.org/10.1109/ACCESS.2019.2960542.

Khan I, Haleem A, Javaid M. Analysing COVID-19 pandemic through cases, deaths, and recoveries. J Oral Biol Craniof Res. 2020;10:450 69. https://doi.org/10.1016/j.jobcr.2020.08.003.

Khurshid A. Applying blockchain technology to address the crisis of trust during the COVID-19 pandemic. JMIR Med Inform. 2020;8: e20477. https://doi.org/10.2196/20477.

Kuo L. Fury in China as footage appears to show officials taking doctors' face masks. The Guardian. 2020. [cited 2020 February 2]. Available from: https://www.theguardian.com/world/2020/feb/02/shamelessoutrage-china-coronavirus-outbreak-mask.

Kuo T-T, Kim H-E, Ohno-Machado L. Blockchain distributed ledger technologies for biomedical and health care applications. J Am 
Med Inform Assoc. 2017;24:1211-20. https://doi.org/10.1093/ jamia/ocx068.

Lacina L. What's needed now to protect health workers: WHO COVID19 briefing. World Economic Forum. 2020. [cited 2020 April 10]. Available from: https:/www.weforum.org/agenda/2020/04/10 april-who-briefing-health-workers-covid-19-ppe-training/.

Mishra D, Haleem A, Javaid M. Analysing the behaviour of doubling rates in 8 major countries affected by COVID-19 virus. J Oral Biol Craniofac Res. 2020;10:478-83. https://doi.org/10.1016/j.jobcr. 2020.08.007.

Nasdaq. VeChain announces blockchain vaccine tracing solution for China. Distributed. 2018. Available from: https://www.nasdaq. com/articles/vechain-announces-blockchain-vaccine-tracingsolution-china-2018-08-16.

OECD Economic Outlook, Interim Report March 2020: coronavirus: the world economy at risk. Organisation for Economic Co-Operation and Development (OECD). 2020. [cited 2020 May 21]. Available from: https://www.oecd.org/economic-outlook/.

Papadopoulos T, Baltas KN, Balta ME. The use of digital technologies by small and medium enterprises during COVID-19: implications for theory and practice. Int J Inf Manag. 2020;2020:102192. https://doi. org/10.1016/j.ijinfomgt.2020.102192.

Powers B. Privacy advocates are sounding alarms over coronavirus surveillance. Coindesk. 2020. [cited 2020 October 4]. Available from: https://www.coindesk.com/privacy-advocates-are-soundingalarms-over-coronavirus-surveillance.

Pratap Singh R, Javaid M, Haleem A, Vaishya R, Ali S. Internet of medical things (IoMT) for orthopaedic in COVID-19 pandemic: roles, challenges, and applications. J Clin Orthop Trauma. 2020;11:713-7. https://doi.org/10.1016/j.jcot.2020.05.011.

Rab S, Javaid M, Haleem A, Vaishya R. Face masks are new normal after COVID-19 pandemic. Diabetes Metab Syndr Clin Res Rev. 2020;14:1617-9. https://doi.org/10.1016/j.dsx.2020.08.021.

Resiere D, Resiere D, Kallel H. Implementation of medical and scientific cooperation in the Caribbean using blockchain technology in coronavirus (Covid-19) pandemics. J Med Syst. 2020;44:123. https:// doi.org/10.1007/s10916-020-01589-4.

Shams SA, Haleem A, Javaid M. Analyzing COVID-19 pandemic for unequal distribution of tests, identified cases, deaths, and fatality rates in the top 18 countries. Diabetol Metab Syndr. 2020;14:95361. https://doi.org/10.1016/j.dsx.2020.06.051.

Sharma TK. Blockchain can track the deadly coronavirus. Blockchain Council 2020. Available from: https://www.blockchain-council. org/blockchain/blockchain-can-track-the-deadly-coronavirus/.
Sharma A, Bahl S, Bagha AK, Javaid M, Shukla DK, Haleem A. Multiagent system applications to fight COVID-19 pandemic. Apollo Med. 2020;17:S41-3. https://doi.org/10.4103/am.am_54_20.

Singh RP, Javaid M, Haleem A, Suman R. Internet of things (IoT) applications to fight against COVID-19 pandemic. Diabetes Metab Syndr Clin Res Rev. 2020;14:521-4. https://doi.org/10.1016/j.dsx.2020. 04.041.

Singh RP, Javaid M, Kataria R, Tyagi M, Haleem A, Suman R. Significant applications of virtual reality for COVID-19 pandemic. Diabetes Metab Syndr Clin Res Rev. 2020;14:661-4. https://doi. org/10.1016/j.dsx.2020.05.011.

Suman R, Javaid M, Haleem A, Vaishya R, Bahl S, Nandan D. Sustainability of coronavirus on different surfaces. J Clin Exp Hepatol. 2020;10:386-90. https://doi.org/10.1016/j.jceh.2020.04. 020.

Ting DSW, Carin L, Dzau V, Wong TY. Digital technology and COVID19. Nat Med. 2020;26:459-61. https://doi.org/10.1038/s41591-0200824-5.

Vaishya R, Bahl S, Singh RP. Letter to the editor in response to: telemedicine for diabetes care in India during COVID19 pandemic and national lockdown period: guidelines for physicians. Diabetes Metab Syndr Clin Res Rev. 2020;14:687-8. https://doi.org/10. 1016/j.dsx.2020.05.027.

Vaishya R, Haleem A, Vaish A, Javaid M. Emerging technologies to combat the COVID-19 pandemic. J Clin Exp Hepatol. 2020;10: 409-11. https://doi.org/10.1016/j.jceh.2020.04.019.

Vaishya R, Javaid M, Khan IH, Haleem A. Artificial intelligence (AI) applications for COVID-19 pandemic. Diabetes Metab Syndr Clin Res Rev. 2020;14:337-9. https://doi.org/10.1016/j.dsx.2020.04. 012.

Waltz E. Big data helps Taiwan fight coronavirus. IEEE Spectrum. 2020. [cited 2020 October 2]. Available from: https://spectrum.iee.org/ the-human-os/biomedical/devices/big-data-helps-taiwan-fightcoronavirus.

$\mathrm{Yu}$ E. Alibaba Cloud offers AI platform to support coronavirus medical efforts. ZDNet. 2020. [cited 2020 October 2]. Available from: https://www.zdnet.com/article/alibaba-cloud-offers-ai-platform-tosupport-coronavirus-medical-research/.

ZIGURAT: Innovation and technology business school. Blockchain in healthcare: the case of coronavirus. 2020. Available from: https:// www.e-zigurat.com/innovation-school/blog/blockchain-inhealthcare/.

Publisher's note Springer Nature remains neutral with regard to jurisdictional claims in published maps and institutional affiliations. 